\title{
A preventive control strategy for static voltage stability based on an efficient power plant model of electric vehicles
}

\author{
Mingshen WANG, Yunfei MU ( $₫)$, Hongjie JIA, \\ Jianzhong WU, Xiuping YAO, Xiaodan YU, \\ Janaka EKANAYAKE
}

\begin{abstract}
With the increasing integration of wind farms and electric vehicles (EVs) in power systems, voltage stability is becoming more and more serious. Based on vehicle-to-grid (V2G), an efficient power plant model of EVs (E-EPP) was developed to estimate EV charging load with available corresponding response capacity under different charging strategies. A preventive control strategy based on E-EPP was proposed to maintain the static voltage stability margin (VSM) of power system above a predefined security level. Two control modes were used including the disconnection of EV charging load ('V1G' mode) and the discharge of stored battery energy back to power grid ('V2G' mode). A modified IEEE 14-bus system with high penetration of wind power and EVs was used to verify the effectiveness of preventive control strategy. Simulation results showed that the proposed strategy can not only improve the static voltage stability of power system with considerable wind generation, but also guarantee the travelling comfort for EV owners.
\end{abstract}

Keywords Electric vehicle (EV), Vehicle-to-grid (V2G), Efficient power plant (EPP), Preventive control, Static voltage stability

CrossCheck date: 15 December 2014

Received: 7 October 2014/Accepted: 10 December 2014/Published online: 21 January 2015

(C) The Author(s) 2015. This article is published with open access at Springerlink.com

M. WANG, Y. MU, H. JIA, X. YU, Key Laboratory of Smart Grid of Ministry of Education, Tianjin University,

Tianjin 300072, China

$(\bowtie)$ e-mail: yunfeimu@tju.edu.cn

J. WU, J. EKANAYAKE, School of Engineering, Institute of

Energy, Cardiff University, Wales CF10 3BN, UK

$\mathrm{X}$. YAO, State Grid Xinjiang Electric Power Company,

Urumqi 830002, China

\section{Introduction}

The development of "low-carbon economy" is drawing more and more attention around the world considering the environment and energy. As a consequence, a number of countries have taken specific initiatives to decarbonize their electrical power system and transport sectors [1-4]. In $\mathrm{UK}$, it is anticipated that a large proportion of renewable energy is wind energy. There may be up to $30 \mathrm{GW}$ wind generation of $100 \mathrm{GW}$ total generation capacity serving around $60 \mathrm{GW}$ load by 2020 [5, 6]. In order to realize the target of reducing emissions from domestic transport sector by $14 \%$ by 2020 , the UK government has supported EV trials with the anticipation that EVs will play a major role in future transport sector [7-9].

According to the large scale penetration of renewable energy generation from intermittent resources, especially wind power, it is fundamental that the electric system is able to appropriately compensate the effects on the variability and randomness of wind energy. Among all the issues caused by the intermittency of wind power, voltage stability is a main constraint for the further development of low-carbon power system $[10,11]$.

A lot of studies have been carried out to analyze the voltage stability integrated with wind generation. The impact of wind power with high penetration on the voltage stability of power system was analyzed in $[12,13]$. A strategy was proposed in [14] to coordinate the reactive power of variable-speed wind generators and other reactive controllers for static voltage stability enhancement which may limit the output of wind power. Battery energy storage system (BESS) was used for regulating the wind power variation to improve the voltage stability of power system [15]. However, as BESS is expensive, its economic benefits for wide deployment are still in question. Although the application of static synchronous compensator can improve 
the voltage stability of power system with wind farms, large scale adoption of these kinds of devices is not economical and practical until now [16-18].

With the support from modern communication and control system, EVs can not only act as a rapid response load of power system, but also support the security operation of power system as a new kind of energy storage system [19]. A real-time scheduling method of EV charging load was proposed to increase voltage security margin in a low-voltage distribution system, but the energy stored in EV batteries was not fully utilized [20]. In [21], EVs were used to provide the voltage support of power system with the integration of photovoltaic power generation, which demonstrates the feasibility of using EVs for voltage control with intermittent renewable energy. However, these studies are unable to fully consider the transportation behaviors of EV owners, which lead to a spatial and temporal distribution of EV charging load and available response capability.

In this paper, an efficient power plant of EVs (E-EPP) was developed to determine the maximum/minimum available response capacity of EVs from a spatial and temporal perspective, considering the travelling comfort of EV owners. A preventive control strategy based on E-EPP was developed to fully utilize the vehicle-to-grid (V2G) capability of EV aggregation along a day, which can not only effectively improve the static voltage stability, but also reduce the emission of carbon dioxide by supporting the integration of wind farms in a power system. The rest of paper is organized as follows. The framework of preventive control strategy is introduced in Sect. 2. Section 3 develops E-EPP model considering the travelling behaviors of EV owners. The preventive control strategy is given in Sect. 4. In Sect. 5, simulations are carried out on a modified IEEE 14-bus system integrated with the high penetration of EVs and wind generation to verify the effectiveness of proposed preventive control strategy. The reduction of carbon dioxide emission is calculated. Conclusions are summarized in Sect. 6.

\section{Framework of integrating EVs for preventive control}

\subsection{Traditional preventive control for static voltage stability}

In conventional power systems, when load demand approaches or even exceeds its transfer limit, voltage instability/collapse happens at the heavy-duty point. With the integration of large wind farms, the transmission mode of power system may be changed on the receiving end. The intermittent output may cause transmission congestion problems. Besides, while sending out active power, wind farms usually absorb considerable reactive power from power system [22]. However, wind power will cause significant impact on voltage stability.

In order to analyze the voltage stability, $S_{\mathrm{GL}}=S_{\mathrm{G}} \cup \boldsymbol{S}_{\mathrm{L}}$ is used as the power injection vector; $\boldsymbol{S}_{\mathrm{G}}=\left(\boldsymbol{P}_{\mathrm{Gc}} \cup \boldsymbol{Q}_{\mathrm{Gc}}\right) \cup$ $\left(\boldsymbol{P}_{\mathrm{Gw}} \cup \boldsymbol{Q}_{\mathrm{Gw}}\right)$ is the corresponding power injection vector of conventional generators and wind farms; $\boldsymbol{S}_{\mathrm{L}}=\boldsymbol{P}_{\mathrm{L}} \cup \boldsymbol{Q}_{\mathrm{L}}$ is the power injector of loads; $\boldsymbol{P}_{\mathrm{Gc}}$ and $\boldsymbol{Q}_{\mathrm{Gc}}$ are the active and reactive power injection vectors of conventional generators, respectively; $\boldsymbol{P}_{\mathrm{Gw}}$ and $\boldsymbol{Q}_{\mathrm{Gw}}$ are the active and reactive power injection vectors of wind farms, respectively. The operating status $\boldsymbol{x}$ is determined by $\boldsymbol{S}_{\mathrm{GL}}$ as described as

$\left\{\begin{array}{l}\boldsymbol{f}\left(\boldsymbol{x}, S_{\mathrm{GL}}\right)=\mathbf{0} \\ \boldsymbol{g}\left(\boldsymbol{x}, S_{\mathrm{GL}}\right) \leq \mathbf{0}\end{array}\right.$

where $\boldsymbol{f}$ is the load flow equation; $\boldsymbol{g}$ is the system operating constrain equation. If $\boldsymbol{x}$ yielded by (1) also satisfies (2), the system is said to be static voltage stability. While, if $\boldsymbol{x}$ satisfies (1) as well as (3), the system is at the critical point of static voltage stability.

$\operatorname{det}\left(f_{x}\right) \neq 0$

$\operatorname{det}\left(\boldsymbol{f}_{x}\right)=0$

where $f_{x}$ is the Jacobian matrix of load flow equation. $\lambda$ is introduced to evaluate static voltage stability margin (VSM), which can be determined by continuous power flow (CPF) according to the direction of load growth and generation dispatch mode $[23,24]$. In order to prevent voltage stability, retaining large enough $\lambda$ under different contingencies is necessary.

Preventive control is a main means to maintain power system operating above a minimum voltage stability margin $\left(\lambda_{\mathrm{cr}}\right)$ under the increased uncertainty brought on by the integration of intermittent renewable generation, such as wind power. Usually, the traditional preventive control of power system for voltage control is divided into three stages, where if the previous stage is effective, the latter stage can be avoided.

Stage I: Adjusting the generator terminal voltage, shunts, and on-load tap changer.

Stage II: Considering the re-dispatching generator active power.

Stage III: Dispatching the load shedding.

The arrangement of above three stages aims to guarantee an uninterruptible power supply to end-users while taking the cost of control into account. Therefore, load-shedding is traditionally regarded as the last means of avoiding voltage collapse. However, this situation has been significantly changed as the V2G capability from EVs was introduced. Active participation based on the voluntary of EV owners will show lower control cost and higher 
efficiency, which can be used as an efficient power plant to participate in stage II for voltage control. In this paper, a preventive control strategy for static voltage stability using $\mathrm{EVs}$ is investigated. It is assumed that the capacity of stage I has been ran out, thereby only the capability of E-EPP provides static voltage control requirements.

\subsection{Framework of preventive control using E-EPP}

The framework of preventive control strategy based on E-EPP is shown in Fig. 1.

Power system dispatch center (PSDC) provides a valuable system-level metric for the minute-to-minute decision to ensure service consistency. One role of PSDC is to obtain the static voltage stability margin $(\lambda)$ associated with the current operating point (distance to voltage collapse), and compute credible contingencies with respect to predefined load directions. CPF is used to compute the VSM of power system [25]. Also, the preventive control strategy is implemented in PSDC for static voltage control of power system.

E-EPP is an aggregate model of a large scale of geographically dispersed EVs connected to power system with
V2G power electronic interface. Although the available response power capacity of a single EV is relatively small, the aggregate capacity of all EVs managed by E-EPP is considerable. E-EPP can serve as an intermediary between EVs and PSDC. On one hand, E-EPP estimates EV charging load with the corresponding available response capacity under different charging strategies. On the other hand, E-EPP is responsible for managing EVs charging/ discharging process of voltage control considering the travelling comfort level of EV owners.

When a voltage contingency happens, E-EPP receives the control signals from PSDC. According to the preventive control strategy, part of EV charging loads are shed from power system ('V1G') or even discharge the stored energy back to grids ('V2G'). Battery state-of-charge (SOC) is used as an indicator for E-EPP to select EV candidates for responding the control signals given by preventive control strategy considering the travelling preference of EV owners. The battery SOC information is obtained via smart meter (SM) and charging point management system (CPM). Then the SOC information flow is transmitted to E-EPP control center for preventive control.

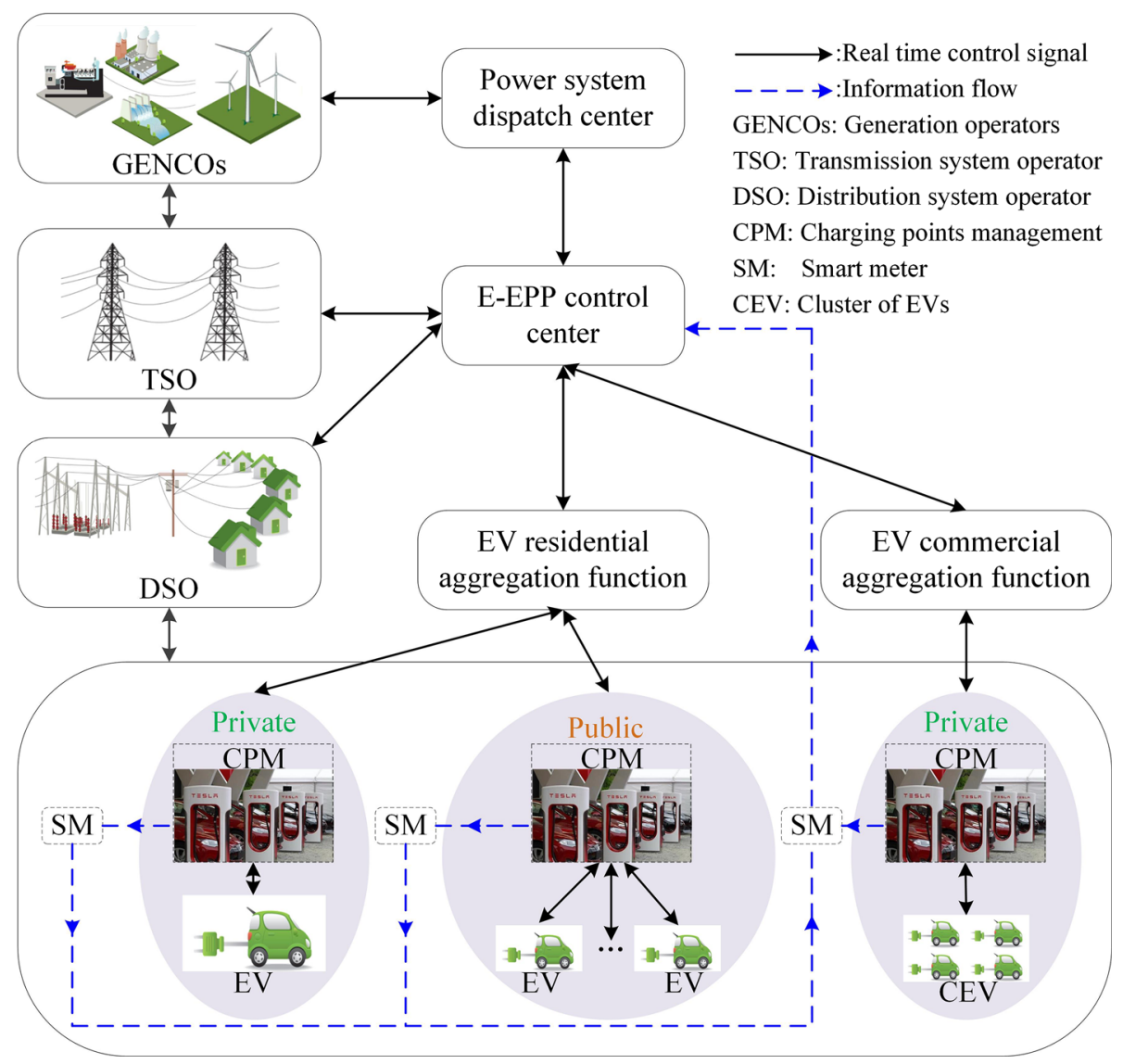

Fig. 1 Integration of EVs for preventive control based on E-EPP 


\section{Efficient power plant model of aggregate EVs}

\subsection{A generic battery model for EVs}

A generic battery model (GBM) is firstly developed to describe the charging/discharging characteristics of various EV batteries. As shown in Fig. 2, GBM is a simple controlled voltage source in series with a constant resistance. The model uses only SOC as a state variable in order to accurately reproduce the manufacturers' curves for four major types of batteries (lead-acid, lithium-ion, nickelcadmium and nickel-metal-hybrid batteries), which are commonly considered as the most promising EV batteries in the future EV market [26].

The battery terminal voltage $V_{\text {batt }}$ is described as

$V_{\text {batt }}=E_{0}-\frac{K}{K_{\mathrm{SOC}}}+A \exp \left(-B \int_{0}^{t} I \mathrm{~d} t\right)-R I$

$K_{\mathrm{SOC}}=1-\int_{0}^{t} I(t) \mathrm{d} t / Q$

where $K_{\mathrm{SOC}}$ is the value of SOC; $E_{0}$ is the constant battery voltage; $K$ is the polarisation voltage; $Q$ is the battery capacity; $I$ is the charging current; $A$ is the exponential zone amplitude; $B$ is the exponential zone time constant inverse; $R$ is the internal resistance.

Assuming a constant charging current $I$ to simplify the integral part, the charging power $P$ is shown as

$P=E_{0} I-\frac{K I}{K_{\mathrm{SOC}}}+A I \exp (-B I t)-R I^{2}$

There are only a few parameters in the proposed GBM. Once the parameters of battery type and capacity are determined, SOC and charging power can be obtained in real time by using this GBM.

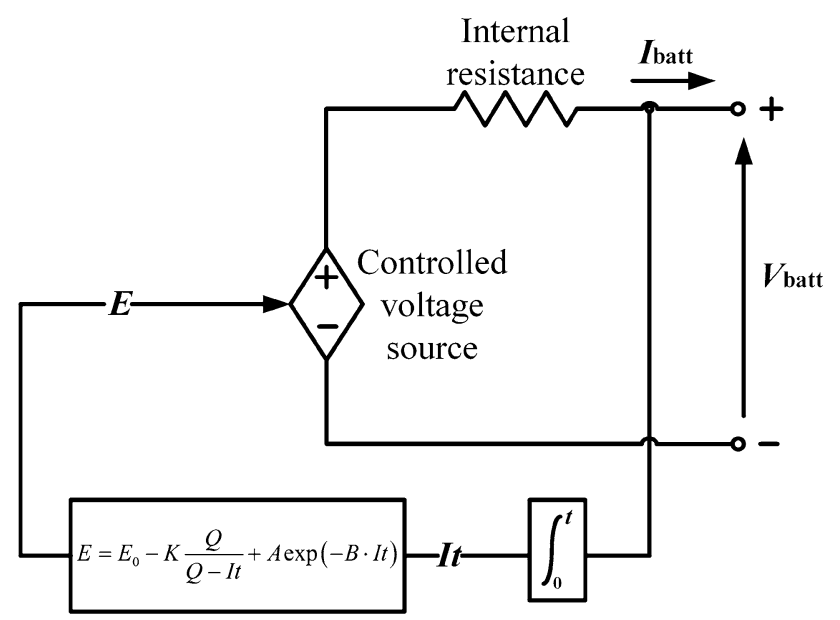

Fig. 2 The generic battery model of EV batteries

\subsection{Formulation of E-EPP}

Considering the travelling behaviors of EV owners, E-EPP is established to evaluate the response capacity of EVs along a day for voltage control. Several uncertainties are considered in this formulation, such as battery type, capacity, energy consumption, daily travelling distance, travelling time and the minimum desired battery SOC for travelling requirement.

\subsubsection{EV classification}

In the formulation of E-EPP, EVs are classified into the following two groups.

Based on the use of transportation, EVs are classified into three types, i.e., home-based-work (HBW), home-based-other (HBO) and non-home-based (NHB). Their proportions in UK are $61 \%, 30 \%$ and $9 \%$, respectively [27]. This classification is used to obtain EV daily travelling distance and time.

According to vehicle types, EVs are classified into four types, i.e., L7e, M1, N1 and N2, and their proportions are $1.49 \%, 87.51 \%, 10 \%$ and $1 \%$, respectively [28]. This classification is used to obtain the battery type, capacity and energy consumption per kilometer of a single EV.

\subsubsection{Battery type, capacity and energy consumption}

Based on the market survey data of various battery types $\left(B_{\mathrm{t}}\right)$ in $\mathrm{UK}$, the proportions of four promising EV batteries, i.e., lead-acid batteries, lithium-ion batteries, nickel-cadmium batteries and nickel-metal-hybrid batteries, are $20 \%, 50 \%, 10 \%$ and $20 \%$, respectively [28]. According to the type of EVs, the distributions of battery capacities $\left(Q_{\mathrm{v}}\right)$ are shown in Table 1 [29], and the parameters in the distributions are further defined by (7) of Gamma distribution and (8) of normal distribution.

$g\left(Q_{v} ; \alpha, \beta\right)=\frac{1}{\beta^{\alpha} \Gamma(\alpha)} Q_{v}^{\alpha-1} \exp \left(-\frac{Q_{v}}{\beta}\right)$

$f\left(Q_{v} ; \mu, \sigma\right)=\frac{1}{\sqrt{2 \pi} \sigma} \exp \left(-\frac{\left(Q_{v}-\mu\right)^{2}}{2 \sigma^{2}}\right)$

Meanwhile, the distributions of EV energy consumption $\left(C_{\mathrm{e}}\right)$ are shown in Table 2 [28].

Table 1 Distributions of EV battery capacities

\begin{tabular}{lllcc}
\hline Type & Distribution & Parameters & Min $(\mathrm{kWh})$ & Max $(\mathrm{kWh})$ \\
\hline L7e & Gamma & $\alpha=10.8 ; \beta=0.8$ & 3.0 & 15.0 \\
M1 & Gamma & $\alpha=4.5 ; \beta=6.7$ & 10.0 & 72.0 \\
N1 & Normal & $\mu=23.0 ; \sigma=9.5$ & 9.6 & 40.0 \\
N2 & Normal & $\mu=85.3 ; \sigma=28.0$ & 51.0 & 120.0 \\
\hline
\end{tabular}




\subsubsection{Daily travelling distance and time}

Based on the use of transportation (HBW, HBO and NHB), daily travelling distance $d$ follows a normal distribution, which is expressed as

$f\left(d ; \mu_{d}, \sigma_{d}\right)=\frac{1}{\sqrt{2 \pi} \sigma_{d}} \exp \left(-\frac{\left(d-\mu_{d}\right)^{2}}{2 \sigma_{d}^{2}}\right)$

where $\mu_{d}$ is the mean daily travelling distance; $\sigma_{d}$ is the standard deviation. For HBW and HBO vehicles, $\mu_{d}=35.9 \mathrm{~km}, \sigma_{d}=19.6 \mathrm{~km}$. While for NHB vehicles, $\mu_{d}=87.1 \mathrm{~km}$, and $\sigma_{d}=24.5 \mathrm{~km} \mathrm{[27].}$

Daily travelling time has close relationship with the transportation behaviors of EV owners. The distributions of starting travelling time $\left(t_{\mathrm{s}}\right)$ and finishing travelling time $\left(t_{\mathrm{f}}\right)$ are shown in Fig. 3 [29].

\subsubsection{Formulation of E-EPP}

1) Determine $B_{\mathrm{t}}, C_{\mathrm{e}}$ and $Q_{\mathrm{v}}$ of a single $\mathrm{EV}$

For a single EV, a Monte Carlo simulation (MCS) method is used to obtain $B_{\mathrm{t}}$ based on the proportion of previous four EV batteries, also $C_{\mathrm{e}}$ and $Q_{\mathrm{v}}$ depending on the distributions in Tables 1 and 2, respectively.

2) Obtain the parameters in GBM

It is assumed that EV battery charging process is reversible. The power sent back to grid by an EV is assumed as a constant while providing voltage control, and it is equal to the power absorbed by the same $\mathrm{EV}$ before controlling. Once $B_{\mathrm{t}}$ and $Q_{\mathrm{v}}$ were determined in step 1, the other parameters in GBM are deduced from the discharging curves supplied by manufacturers [26]. Then the charging process consisting of charging power $(P)$ and SOC is obtained via GBM.

3) Determine $d$ and charging starting time

The daily travelling distance is obtained by (9) with a MCS process. The charging time $t_{\mathrm{sc}}$ of an EV battery is determined by daily transportation behavior and the charging strategies. Three charging strategies are considered in the formulation of E-EPP.

Table 2 Distributions of energy consumption per kilometer

\begin{tabular}{lllll}
\hline$C_{\mathrm{e}}(\mathrm{kWh} / \mathrm{km})$ & $\mathrm{L} 7 \mathrm{e}$ & $\mathrm{M} 1$ & $\mathrm{~N} 1$ & $\mathrm{~N} 2$ \\
\hline $0.05-0.10$ & 0.34 & 0.05 & 0.00 & 0.00 \\
$0.10-0.15$ & 0.58 & 0.35 & 0.29 & 0.00 \\
$0.15-0.20$ & 0.08 & 0.45 & 0.14 & 0.00 \\
$0.20-0.25$ & 0.00 & 0.15 & 0.57 & 0.00 \\
$0.45-0.50$ & 0.00 & 0.00 & 0.00 & 0.50 \\
$0.50-0.55$ & 0.00 & 0.00 & 0.00 & 0.25 \\
$0.80-0.85$ & 0.00 & 0.00 & 0.00 & 0.25 \\
\hline
\end{tabular}

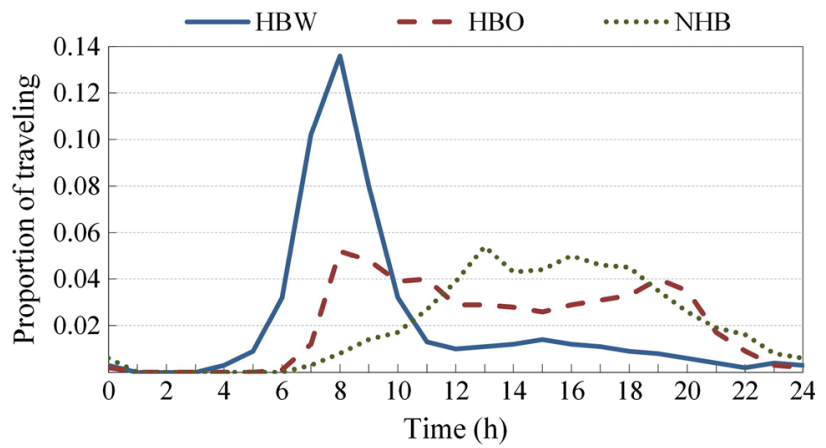

(a) Starting time

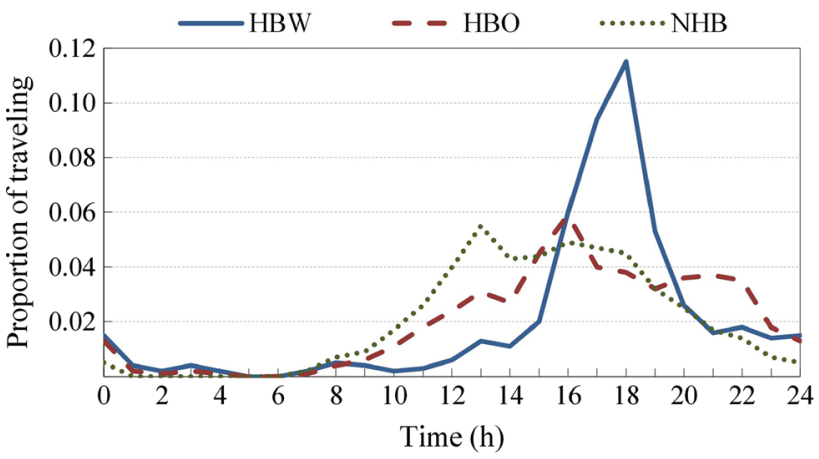

(b) Finishing time

Fig. $3 \mathrm{EV}$ travelling time distributions of $\mathrm{HBW}, \mathrm{HBO}$ and $\mathrm{NHB}$

a. Dumb Charging:

In dumb charging, it is assumed that all EVs start to charge as soon as their daily trips are finished. Therefore, $t_{\mathrm{sc}}$ is equal to $t_{\mathrm{f}}$ which is determined by the distributions shown in Fig. 3b.

b. Smart Charging:

It is envisaged that there will be an active management system based on two hierarchical control structures by an Aggregator and the system operators (TSO/DSO), respectively. Furthermore, it is assumed that EV charging is controlled according to the Aggregator's market negotiations or the need of system operators. Smart charging is described by (10) with $\mu_{\mathrm{sc}}(1: 00)$ and $\sigma_{\mathrm{sc}}(5 \mathrm{~h})$ [29]. Compared with "dumb" charging, the model of "smart" charging represents the shift of EV charging load from the system peak demand time to the valley hours.

$f\left(t_{s c} ; \mu_{s c}, \sigma_{s c}\right)=\frac{1}{\sqrt{2 \pi} \sigma_{s c}} \exp \left(-\frac{\left(t_{s c}-\mu_{s c}\right)^{2}}{2 \sigma_{s c}^{2}}\right)$

c. Hybrid Charging:

Hybrid charging is a charging strategy with $50 \%$ of the EVs adopting dumb charging and the remaining $50 \%$ following smart charging.

4) Determine the initial SOC when an EV starts to charge 
Assuming SOC drops linearly with the travelling distance [27], $K_{\mathrm{SOC}_{0}}$ is determined as

$K_{\mathrm{SOC}_{0}}=\left(\delta-\frac{d}{d_{\mathrm{t}}}\right) \times 100 \%$

where $K_{\mathrm{SOC}_{0}}$ is the value of $\mathrm{SOC}_{0} ; d_{\mathrm{t}}$ is the maximum travelling distance and $d_{\mathrm{t}}=Q_{\mathrm{v}} / C_{\mathrm{e}} ; \delta$ is SOC of a single EV before travelling, and it varies uniformly within $[0.8,0.9]$ to maintain the lifetime of a battery [28].

5) Determine the response state of an EV

Assuming a minimum desired SOC $\left(K_{\mathrm{SOC}_{\mathrm{e}}}\right)$ (for satisfying the travelling requirements of $\mathrm{EV}$ owners) is $60 \%$. For an individual $\mathrm{EV}$ of $\mathrm{EV}_{i}$, the charging state at time $t\left(\eta_{i, t}\right)$ is determined by (12). If $\eta_{i, t}$ is 0 , the charging $\mathrm{EV}_{i}$ is not controllable. If $\eta_{i, t}$ is 1 , the charging $\mathrm{EV}_{i}$ can be selected to charge, stop charging ('V1G') or discharging ('V2G'). While if $\eta_{i, t}$ is 2 , the idle $\mathrm{EV}_{i}$ can be selected to discharge of stored battery energy back to power grid ('V2G').

$\eta_{i, t}= \begin{cases}0 & 0<K_{\mathrm{SOC}_{i, t}} \leq K_{\mathrm{SOC}_{\mathrm{e}}} \\ 1 & K_{\mathrm{SOC}_{\mathrm{e}}<K_{\mathrm{SOC}_{i, t}} \leq \delta_{i}} \\ 2 & \delta_{i} \leq K_{\mathrm{SOC}_{i, t}}<100 \%\end{cases}$

6) Based on the MCS process, steps 1-5 are repeated $n$ times for $n$ EVs. The real-time charging power $\left(P_{\mathrm{E}-\mathrm{EPP}, t}\right)$ is obtain by (13). The upper available response boundary $\left(P_{\text {upper }, t}\right)$ and lower available response boundary $\left(P_{\text {lower, } t}\right)$ of E-EPP are given in (14) and (15), respectively.

$P_{\mathrm{E}-\mathrm{EPP}, t}=\sum_{i=1}^{l_{t}} P_{i, t}+\sum_{j=1}^{s_{t}} P_{j, t}$

$P_{\text {upper }, t}=\sum_{i=1}^{l_{t}} P_{i, t}+\sum_{j=1}^{m_{t}} P_{j, t}+\sum_{k=1}^{n_{t}} P_{k, t}$

$P_{\text {lower }, t}=\sum_{i=1}^{l_{t}} P_{i, t}-\sum_{j=1}^{m_{t}} P_{j, t}-\sum_{k=1}^{n_{t}} P_{k, t}$

where $P_{i, t}$ is the charging/discharging power of $\mathrm{EV}_{i}$ at time $t ; l_{t}$ is the number of EVs when $\eta_{i, t}$ is $0 ; m_{t}$ is the number of EVs when $\eta_{i, t}$ is $1 ; s_{t}$ is the number of charging EVs among the $m_{t}$ EVs ( $s_{t}$ is obtained by the EV charging state information of E-EPP control center); $n_{t}$ is the number of EVs when $\eta_{i, t}$ is 2 .

\section{Preventive control strategy based on E-EPP}

In this section, a preventive control strategy for static voltage stability is proposed based on the E-EPP. During a voltage contingency, the available capacity of the E-EPP is fully utilized for voltage control considering the travelling comfort constraints of EV owners. Active Participation
Factor (APF) is used in the preventive control to determine the optimum bus to response to the voltage event.

\subsection{Bus response order based on APF}

In this preventive control strategy, APF is used to determine the optimum load bus response order of power system for voltage control, as depicted in (16) [25].

$\left[\begin{array}{c}\Delta \boldsymbol{P} \\ \Delta \boldsymbol{Q}\end{array}\right]=\left[\begin{array}{ll}\boldsymbol{J}_{\mathrm{P} \theta} & \boldsymbol{J}_{\mathrm{PV}} \\ \boldsymbol{J}_{\mathrm{Q} \theta} & \boldsymbol{J}_{\mathrm{QV}}\end{array}\right]\left[\begin{array}{c}\Delta \boldsymbol{\theta} \\ \Delta \boldsymbol{V}\end{array}\right]$

where $\Delta \boldsymbol{P}$ is the active power variations of the buses; $\Delta \boldsymbol{Q}$ is the reactive power variations of the buses; $\Delta \boldsymbol{\theta}$ is the voltage angle variations of the buses; $\boldsymbol{\Delta} \boldsymbol{V}$ is the voltage magnitude variations of the buses; $\boldsymbol{J}_{\mathrm{P} \theta}, \boldsymbol{J}_{\mathrm{PV}}, \boldsymbol{J}_{\mathrm{Q} \theta}$ and $\boldsymbol{J}_{\mathrm{QV}}$ are Jacobian sub-matrices representing the sensitivities of active and reactive power to voltage angle and magnitude, respectively.

During the CPF process, the Jacobian matrix $\boldsymbol{J}$ is obtained at the critical operating point of the system as

$\boldsymbol{J}_{2 \mathrm{NPQ}+\mathrm{NPV}}=\boldsymbol{\Phi} \boldsymbol{\Lambda} \boldsymbol{\Gamma}$

The first $(\mathrm{NPV}+\mathrm{NPQ})$ elements of the right and left eigenvector associated to the critical eigenvalue of $\boldsymbol{J}$ are defined as APF of all buses in the system. In this paper, EVs charging at load bus with the largest APF will be firstly selected to respond to the voltage events in the preventive control strategy.

As the operation status of power system (such as the load level) fluctuates with time, the load bus response order is changed dynamically.

\subsection{Preventive control strategy based on E-EPP}

The SOC is a key indicator to select the satisfied EVs for static voltage stability response in the preventive control strategy. Considering the minimum desired SOC for travelling of EV owners, EVs with high SOC level will participate in the control strategy firstly. The successive steps of the preventive control strategy are listed as follow.

1) Based on the CPF process, compute the VSM $\left(\lambda_{t}\right)$ of power system in real time.

2) If $\lambda_{t} \geq \lambda_{\mathrm{cr}}$, voltage is stable, calculate $\lambda$ for next time. If $\lambda_{t}<\lambda_{\mathrm{cr}}$, the voltage enters an emergency state, preventive control from EVs is started, go to next step.

3) Obtain the APFs, and queue the load buses by the APF values from high to low. According to the E-EPP model, evaluate the available response capacity of EVs at each load bus under 'V1G' and 'V2G' modes, respectively.

4) If there are existing available ' $\mathrm{V} 1 \mathrm{G}$ ' EVs for preventive control, go to step 5. Otherwise, go to step 6 for 
'V2G'. If EVs for 'V1G' or 'V2G' are unavailable, some emergency methods (such as load shedding) are taken for voltage stability control.

The SOC threshold $l\left(l_{\min } \leq l \leq l_{\max }\right)$ is used to select satisfying EVs with $K_{\mathrm{SOC}} \geq l$ to respond to voltage events, and $l_{\max }\left(l_{\min }\right)$ is the upper (lower) boundary, where $l_{\min }$ is equal to $K_{\mathrm{SOC}_{\mathrm{e}}}$ for satisfying the travelling demand of EV owners.

5) Among all the load buses having connected EVs with $K_{\mathrm{SOC}} \geq l$, determine the bus with the highest APF, and the satisfying EVs connected to this load bus stop charging for preventive control.

If system still exists charging EVs with $K_{\mathrm{SOC}} \geq l$, go to step 1. Otherwise, $l=l-\Delta l \geq l_{\min }$, where $\Delta l=\alpha\left(\lambda_{\text {cr }}-\lambda_{t}\right)$ $(\alpha$ is a control coefficient).

6) Among all the load buses having connected EVs with $K_{\mathrm{SOC}} \geq l$, determine the bus with the highest APF, and the satisfying EVs connected to this load bus discharge stored energy back to grid for preventive control.

If system still exists EVs with $K_{\mathrm{SOC}} \geq l$, go to step 1 . Otherwise, $l=l-\Delta l \geq l_{\min }$, where $\Delta l=\alpha\left(\lambda_{\mathrm{cr}}-\lambda_{t}\right)$.

Taking a system with five load buses (N1, N2, N3, N4 and N5) as an example, and assuming the relative APF value at time $t$ is $\mathrm{N} 1>\mathrm{N} 2>\mathrm{N} 3>\mathrm{N} 4>\mathrm{N} 5$, the 'V1G' or ' $\mathrm{V} 2 \mathrm{G}$ ' response order with a constant $\Delta l=5 \%$ is shown in Fig. 4.

If all the EVs for 'V1G' or 'V2G' service recharge simultaneously after the voltage event, there will be a sudden drop on the system VSM. Therefore, the gradual recharging strategy based on temporal-spatial distribution is also proposed. When the VSM is higher than $\lambda_{\text {high }}$, the EVs will connect to power system for recharging based on the opposite order of preventive control until the VSM is lower than $\lambda_{\text {low }}$, where $\lambda_{\text {high }}>\lambda_{\text {low }}>\lambda_{\text {cr }}$. Taking the same system as an example, the recharging order after preventive control is shown in Fig. 5. As a result, the EV with lower SOC level can be recharged firstly, which can improve the travelling comfort level.

\section{Case study}

In this section, a modified IEEE 14-bus system with wind farms (GW3, GW6 and GW8) is used to illustrate the preventive control strategy, as shown in Fig. 6.

The system is divided into three function zones: residential, commercial and industrial zones. Figure 7 gives the load profiles during one day in different zones, which is provided by UKGDS [30].

It is assumed that the wind farms GW3, GW6 and GW8 are composed of doubly fed generators. A typical output of wind power during one day in UK is shown in Fig. 8 [30].

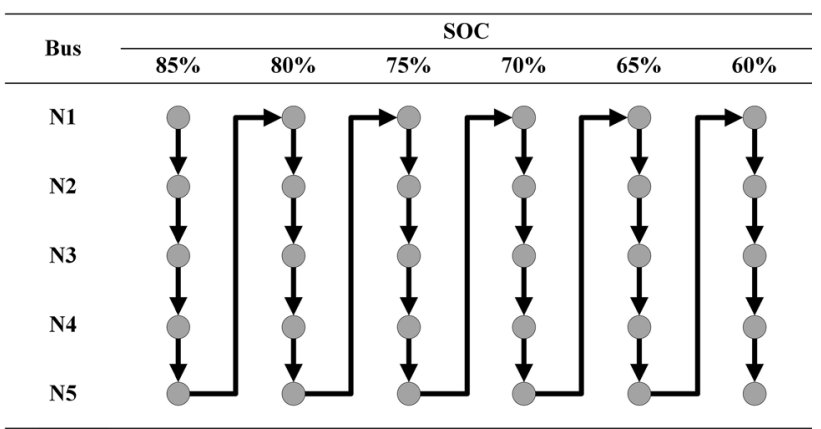

Fig. 4 Response order of 'V1G' or 'V2G' in the preventive control

In this modified IEEE 14-bus system, the peak load is $397.05 \mathrm{MW}$, which is $0.58 \%$ of the UK peak electric demand [31]. The EV numbers in different zones are shown in Table 3 , which also account for $0.58 \%$ of all EVs in UK [32].

Assuming EV charging facilities have been set up, the EV number at each load bus depends on the proportion of its initial load (without EV charging loads). To illustrate the available capacity of aggregate EVs, the E-EPPs under three charging strategies at bus 10 along a day are shown in Fig. 9.

Comparing dumb charging with smart charging, it is clear that the peak charging load is shifted from 18:00 to 01:00. Hybrid charging is a compromised strategy between dumb and smart charging. To compare and analyze these three charging strategies, the VSM $(\lambda)$ is shown in Fig. 10. Under dumb charging, the peak charging load is in coincidence with the initial peak load, and it is easy to see that the system VSM is lower than $\lambda_{\text {cr }}$ from 17:00 to 23:00.

To verify the effectiveness of the preventive control strategy, it is defined that $\lambda_{\mathrm{cr}}=2.5, \quad \lambda_{\text {high }}=3.5$, $\lambda_{\text {low }}=3.3, \quad l_{\min }=60 \%, \quad l_{\max }=85 \%$ and $\alpha=0.12$. Taking dumb charging as an example, the VSM results of a typical day before and after preventive control are shown in Fig. 11.

From 8:00 to 11:00, a line outage happens on line 6-11, and the VSM of power system decreases below $\lambda_{\text {cr }}$ suddenly. As shown in Fig. 9a, the available response capacity

\begin{tabular}{|c|c|c|c|c|c|c|}
\hline \multirow{2}{*}{ Bus } & \multicolumn{6}{|c|}{ SOC } \\
\hline & $85 \%$ & $80 \%$ & $75 \%$ & $70 \%$ & $65 \%$ & $60 \%$ \\
\hline N1 & & & & & & \\
\hline $\mathrm{N} 2$ & & & & & & \\
\hline N3 & & 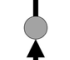 & & & & \\
\hline N4 & & & & & & 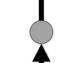 \\
\hline N5 & & & & & & \\
\hline
\end{tabular}

Fig. 5 Recharging order in the preventive control 


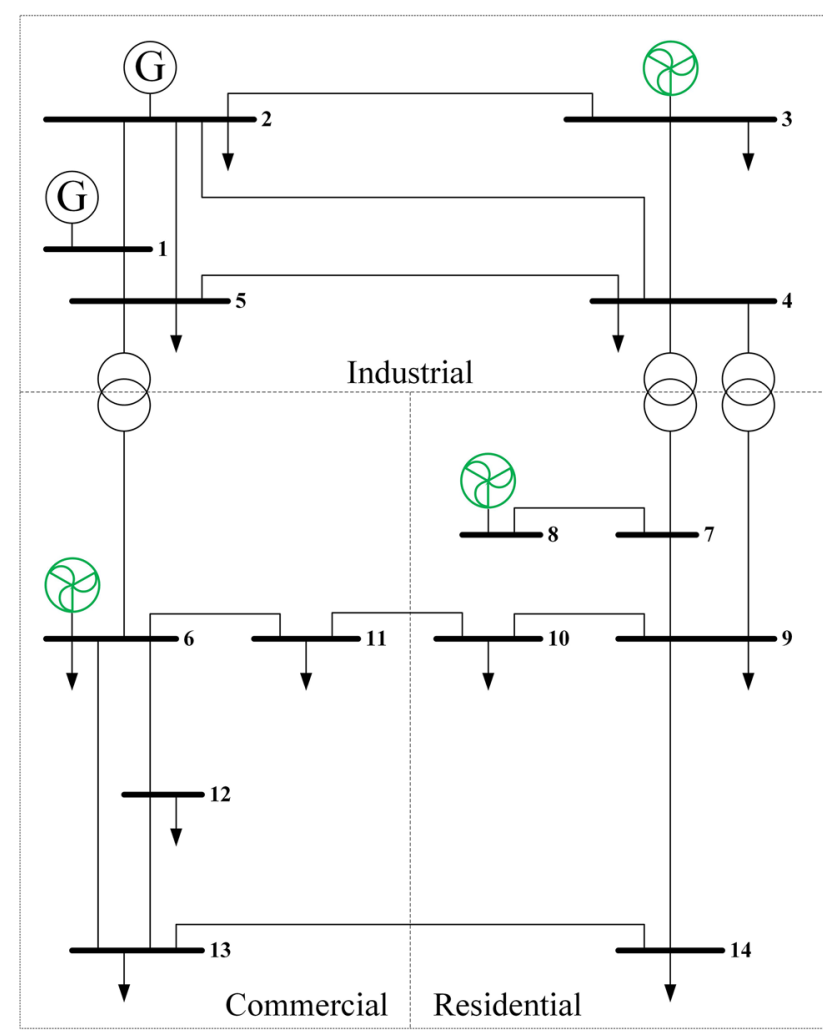

Fig. 6 The modified IEEE-14 bus system

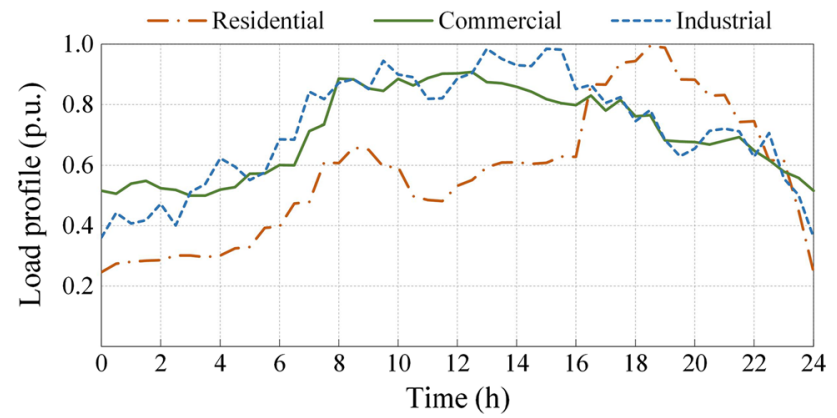

Fig. 7 Load profile of different zones for one day

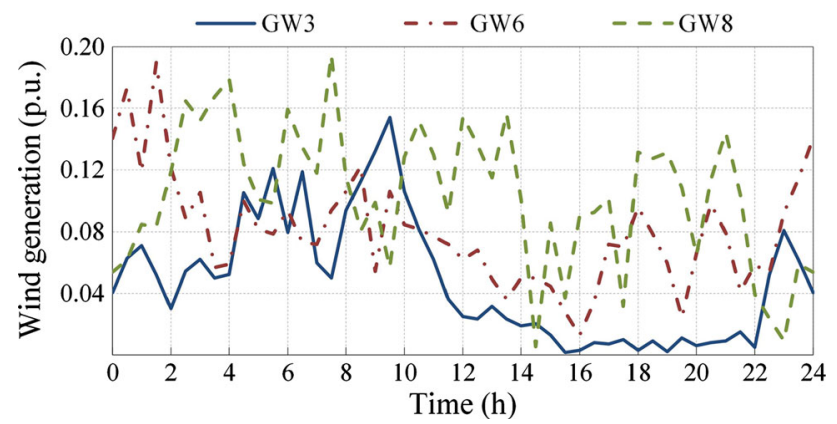

Fig. 8 Wind generation for one day
Table $3 \mathrm{EV}$ numbers for different function zones

\begin{tabular}{lrrrc}
\hline Zones & HBW & HBO & NHB & All EV groups \\
\hline Residential & 100650 & 49500 & 0 & 150150 \\
Commercial & 0 & 0 & 2500 & 2500 \\
Industrial & 0 & 0 & 12400 & 12400 \\
Total & 100650 & 49500 & 14900 & 165050 \\
\hline
\end{tabular}

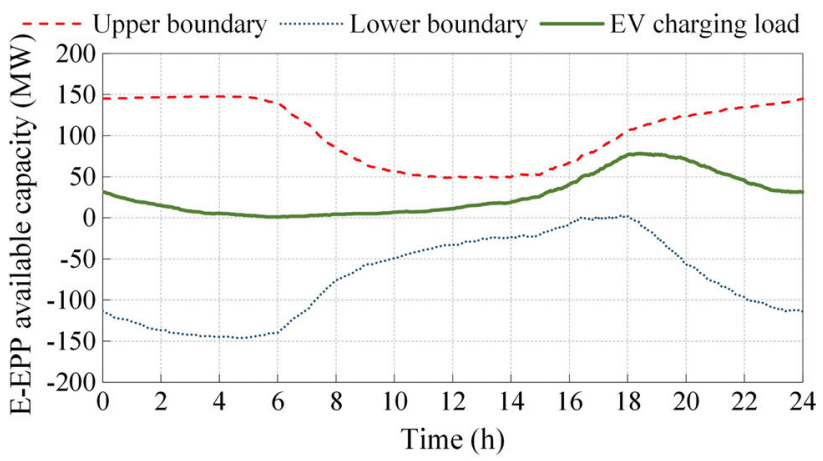

(a) Dumb charging

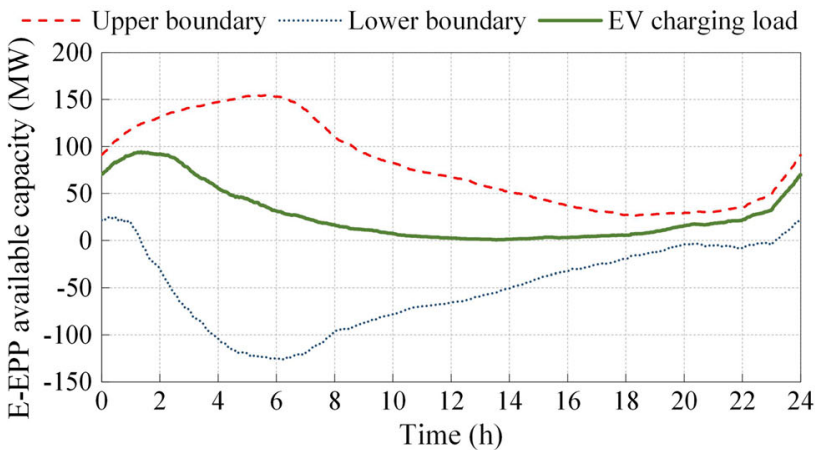

(b) Smart charging

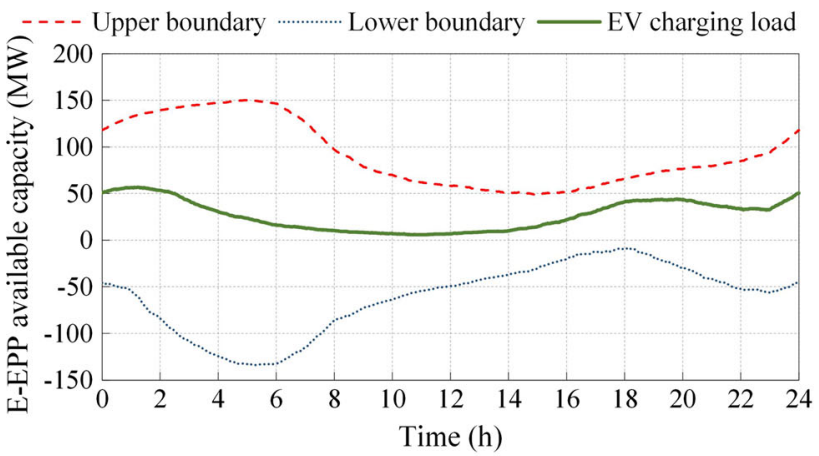

(c) Hybrid charging

Fig. 9 The E-EPP with available response capacity along a day at bus 10

for ' $\mathrm{V} 1 \mathrm{G}$ ' is rather small during this period. But there are EVs which have completed their charging process, and still connecting to the power system. These EVs are available for 'V2G' control. 


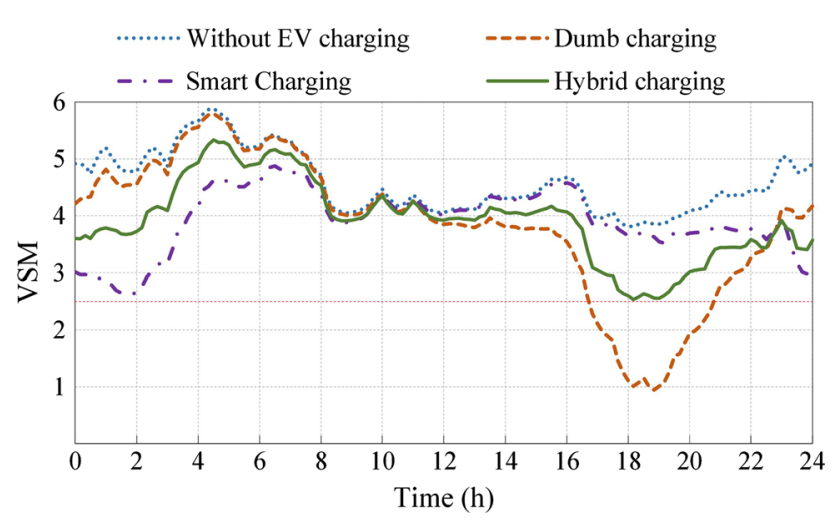

Fig. 10 The VSM along a day under three charging strategies

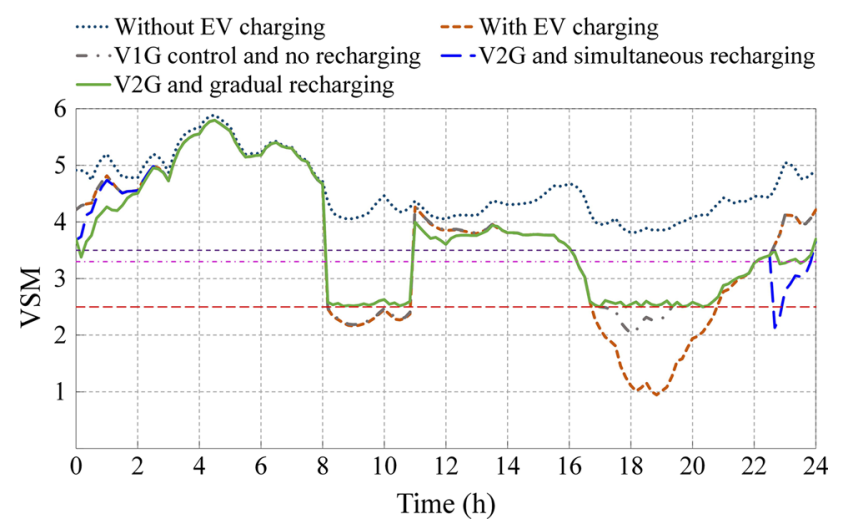

Fig. 11 The preventive control results with dumb charging

While from 17:00 to 21:00, because of a loss of the wind generation and the peak charging load of EVs, the system VSM falls below $\lambda_{\mathrm{cr}}$. Although the charging load of EVs during this period is large, only ' $\mathrm{V} 1 \mathrm{G}$ ' control cannot restore the VSM higher than $\lambda_{\mathrm{cr}}$, as the curve of ' $\mathrm{V} 1 \mathrm{G}$ ' control with no recharging shown in Fig. 11. 'V2G' is further needed for voltage stability control of power system compared with the curve of 'V2G' and gradual recharging in Fig. 11 during this period.

If all the EVs for ' $\mathrm{V} 1 \mathrm{G}$ ' and 'V2G' service recharge simultaneously, there will be a sudden drop of the system VSM at 22:30 as shown in Fig. 11. Under the gradual recharging strategy based on special-temporal distribution, this recharging strategy will ensure smaller fluctuations on voltage as the profile of ' $\mathrm{V} 2 \mathrm{G}$ ' and gradual recharging shown in Fig. 11 during 22:00 to 00:00 and 00:00 to 02:00.

Then to further introduce the process of preventive control strategy, the E-EPP response profile at bus 10 after control is shown in Fig. 12.

As depicted from the above results, using EVs for the preventive control of power system can significantly relieve the static voltage stability issue caused by the wind farms, which can support the integration of wind farms and

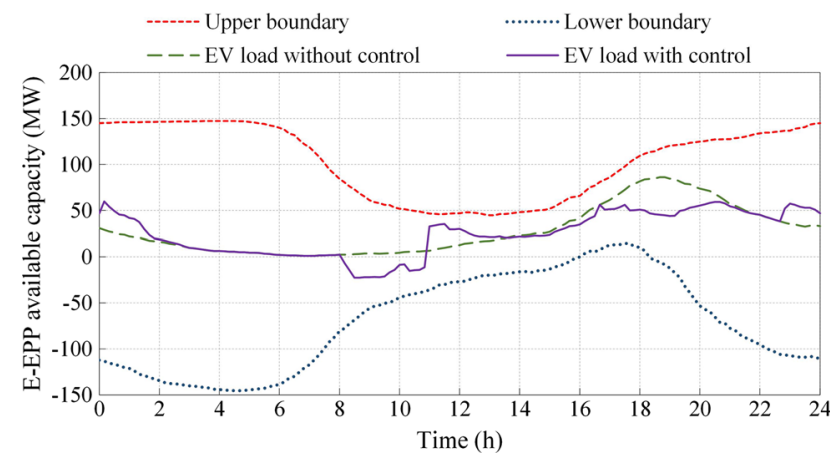

Fig. 12 E-EPP after preventive control

low-carbon economy. For example, according to the carbon dioxide emission factor of thermal power $\left(k_{\mathrm{CO}_{2}}=0.9109 \mathrm{~kg} /(\mathrm{kWh})\right)$ and the calculation method proposed in [33], the emission reduction of carbon dioxide coming from the wind farms at bus 3, 6 and 8 is $506.55 \mathrm{t}$ for a typical day. And this will be of significant importance to the low-carbon economy.

\section{Conclusions}

In this paper, a preventive control strategy from EVs based on E-EPP is proposed to maintain static voltage stability of power system under the V2G concept. The following conclusions are drawn:

1) EVs have great potential to support the voltage control of power system. The developed preventive control strategy can fully utilize the V2G capability of the EV aggregation along a day, which can effectively improve static voltage stability of power system. However, the contribution from EVs has an obvious temporal distribution along a day, which has close relationship with charging strategies.

2) E-EPP is able to determine the maximum/minimum available response capacity of EVs from a spatial and temporal perspective under three charging strategies, considering the travelling comfort levels of EV owners.

3) The gradual recharging strategy can ensure smaller fluctuations on VSM, which can solve the second disturbance problem (caused by sudden increase in EV charging power) on power system.

4) EVs and wind farms have great effect on the emission reduction of carbon dioxide, which can promote the lowcarbon economy to some extent.

Acknowledgments This work was supported in part by the National Natural Science Foundation of China (collaborating with EPSRC of UK) (Nos. 51361130152 and EP/L001039/1), the National Science and Technology Support Program of China (No. 2013BAA01B03), and Research on Reactive Power Control and Comprehensive Evaluation Technique of Large Scale Integration of Wind/Photovoltaic Power Generation (No. NY71-14-035). 
Open Access This article is distributed under the terms of the Creative Commons Attribution License which permits any use, distribution, and reproduction in any medium, provided the original author(s) and the source are credited.

\section{References}

[1] Parkinson S, Wang D, Djilali N (2012) Toward low carbon energy systems: the convergence of wind power, demand response, and the electricity grid. In: Proceedings of the 2012 IEEE international conference on innovative smart grid technologies-Asia (ISGT Asia'12), Tianjin, 21-24 May 2012, 8 pp

[2] Vithayasrichareon P, MacGill IF (2011) Generation portfolio analysis for low-carbon future electricity industries with high wind power penetrations. In: Proceedings of the 2011 IEEE Trondheim PowerTech, Trondheim, 19-23 June 2011, 6 pp

[3] Boulanger AG, Chu AC, Maxx S et al (2014) Vehicle electrification: status and issues. Proc IEEE 99(6):1116-1138

[4] Doolan R, Muntean GM (2014) Reducing carbon emissions by introducing electric vehicle enhanced dedicated bus lanes. In: Proceedings of the 2014 IEEE intelligent vehicles symposium, Dearborn, 8-11 Jun 2014, pp 1011-1016

[5] Jiang LP, Chi YN, Qin HY et al (2011) Wind energy in China. IEEE Power Energy Mag 9(6):36-46

[6] Mu YF, Wu JZ, Ekanayake J et al (2013) Primary frequency response from electric vehicles in the Great Britain power system. IEEE Trans Smart Grid 4(2):1142-1150

[7] Oudalov A, Chartouni D, Ohler C (2007) Optimizing a battery energy storage system for primary frequency control. IEEE Trans Power Syst 22(3):1259-1266

[8] US Environmental Protection Agency (2012) http://www.epa. gov/otaq/climate/documents/420f12063.pdf

[9] Yamashita D, Niimura T, Yoshimi K et al (2012) Optimal strategy to support the development of charging infrastructure for electric vehicles towards low carbon emissions. In: Proceedings of the 2014 IEEE Power and Energy Society General Meeting, San Diego, 22-26 July 2012, 9 pp

[10] Vittal E, O'Malley M, Keane K (2010) A steady-state voltage stability analysis of power systems with high penetrations of wind. IEEE Trans Power Syst 25(1):433-442

[11] Ben-Kilani K, Elleuch M (2013) Structural analysis of voltage stability in power systems integrating wind power. IEEE Trans Power Syst 28(4):3785-3794

[12] Hossain MJ, Pota HR, Mahmud MA et al (2012) Investigation of the impacts of large-scale wind power penetration on the angle and voltage stability of power systems. IEEE Syst J 6(1):76-84

[13] Mi ZQ, Tian HF, Yu Y et al (2009) Study on voltage stability of power grid with large scale wind farm interconnected. In: Proceedings of the 2009 IEEE international conference on sustainable power generation and supply (SUPERGEN'09), Nanjing, 6-7 April 2009, 6 pp

[14] Kumar VSS, Reddy KK, Thukaram D (2014) Coordination of reactive power in grid-connected wind farms for voltage stability enhancement. IEEE Trans Power Syst 29(5):2381-2390

[15] Le HT, Santoso S, Nguyen TQ (2012) Augmenting wind power penetration and grid voltage stability limits using ESS: application design, sizing, and a case study. IEEE Trans Power Syst 27(1):161-171

[16] Arulampalam A, Barnes M, Jenkins N et al (2006) Power quality and stability improvement of a wind farm using STATCOM supported with hybrid battery energy storage. IEE P-Gener Transm Distrib 153(6):701-710
[17] El Moursi MS, Bak-Jensen B, Abdel-Rahman MH (2011) Coordinated voltage control scheme for SEIG-based wind park utilizing substation STATCOM and ULTC transformer. IEEE Trans Sustain Energy 2(3):246-255

[18] Fu R, Sun WP, Yang J et al (2013) Study on application of STATCOM in voltage stability of wind farm incorporated system. In: Proceedings of the 32nd Chinese control conference (CCC'13), Xi'an, 26-28 July 2013, pp 8868-8873

[19] Ma YC, Houghton T, Cruden A et al (2012) Modeling the benefits of vehicle-to-grid technology to a power system. IEEE Trans Power Syst 27(2):1012-1020

[20] Luo X, Chan KW (2014) Real-time scheduling of electric vehicles charging in low-voltage residential distribution systems to minimise power losses and improve voltage profile. IET Gener Transm Distrib 8(3):516-529

[21] Foster JM, Trevino G, Kuss M et al (2013) Plug-in electric vehicle and voltage support for distributed solar: theory and application. IEEE Syst J 7(4):881-888

[22] Mu YF, Jia HJ (2010) An approach to determining the local boundaries of voltage stability region with wind farms in power injection space. Sci China Tech Sci 53(12):3232-3240

[23] Chiang HD, Jin LC, Varghese M et al (2009) Linear and nonlinear methods for contingency analysis in on-line voltage security assessments. In: Proceedings of the 2009 IEEE Power and Energy Society General Meeting, Calgary, 26-30 July 2009, $6 \mathrm{pp}$

[24] Chiang HD, Flueck AJ, Shah KS et al (1995) CPFLOW: a practical tool for tracing power system steady-state stationary behavior due to load and generation variations. IEEE Trans Power Syst 10(2):623-634

[25] Affonso CM, da Silva LCP, Lima FGM et al (2004) MW and MVar management on supply and demand side for meeting voltage stability margin criteria. IEEE Trans Power Syst 19(3):1538-1545

[26] Tremblay O, Dessaint LA, Dekkiche AI et al (2007) A generic battery model for the dynamic simulation of hybrid electric vehicles. In: Proceedings of the 2007 IEEE vehicle power and propulsion conference (VPPC'07), Arlington, 9-12 Sept 2007, pp 284-289

[27] Qian KJ, Zhou CK, Allan M et al (2011) Modeling of load demand due to EV battery charging in distribution systems. IEEE Trans Power Syst 26(2):802-810

[28] Ball R, Keers N, Alexander M et al (2010) Modeling electric storage devices for EV. Project MERGE, WP 2 Task 2.1 Deliverable D2.1

[29] Mu YF, Wu JZ, Jenkins N et al (2014) A spatial-temporal model for grid impact analysis of plug-in electric vehicles. Appl Energy 144:456-465

[30] United Kingdom generic distribution system (UKGDS) (2006) Typical load patterns, DG output data. Distributed Generation and Sustainable Electrical Energy Centre, University of Strathclyde, Glasgow, 2007

[31] National Grid (2010) Metered half-hourly electricity demands. National Grid, London

[32] Transport Statistics Bulletin-Vehicle Licensing Statistics (2010) National statistics. Department for Transport, London

[33] Yu DY, Huang HL, Lei $\mathrm{M}$ et al (2012) $\mathrm{CO}_{2}$ reduction benefit by coordinated dispatch of electric vehicle charging and wind power. Autom Electr Power Syst 36(10):14-18 (in Chinese)

Mingshen WANG is a doctor student majoring in Power System and its Automation in Tianjin University. His main research interests include power system stability analysis, renewable energy integration, and smart grids. 
Yunfei MU is a lecturer of Tianjin University. His special fields of interests include power system stability analysis and control, renewable energy integration and electric vehicle.

Hongjie JIA is a professor of Tianjin University. His special fields of interests include power system stability analysis and control, distribution network planning, renewable energy integration and smart grids.

Jianzhong WU is currently a Reader in Institute of Energy, Cardiff School of Engineering, Cardiff University, UK. His research activities are focused on energy infrastructure and smart grids.

Xiuping YAO is a senior engineer of Xinjiang Electric Power Dispatching and Communication Center. Her special fields of interests include power system stability analysis.
Xiaodan YU is now a lecture of Tianjin University. Her special fields of interests include power system stability analysis, nonlinear dynamic circuit.

Janaka EKANAYAKE is a professor of Peradeniya University, and a senior lecturer in Institute of Energy, Cardiff School of Engineering, Cardiff University, UK. His main research interests include power electronic applications for power systems, renewable energy generation and its integration. He is also a fellow of IET. 